\title{
Prospectively assessing risk for premature ovarian senescence in young females: a new paradigm
}

\author{
Norbert Gleicher ${ }^{1,2^{*}}$, Vitaly A Kushnir ${ }^{1}$ and David H Barad ${ }^{1,2}$
}

\begin{abstract}
Background: Approximately 10\% of women suffer from premature ovarian senescence (POS), ca. $9 \%$ as occult primary ovarian insufficiency (OPOI, also called premature ovarian aging, $\mathrm{POA}$ ) and ca. $1 \%$ as primary ovarian insufficiency (POI, also called premature ovarian failure, POF). In a large majority of cases POS is currently only diagnosed at advanced clinical stages when women present with clinical infertility.
\end{abstract}

Methods: We here, based on published evidence, suggest a new diagnostic paradigm, which is based on identifying young women at increased risk for POS at much earlier stages.

Results: Risk factors for POS are known from the literature, and can be used to identify a sub-group of young women at increased risk, who then are followed sequentially with serial assessments of functional ovarian reserve (FOR) until a diagnosis of POS is either reached or refuted. At approximately $25 \%$ prevalence in general U.S. populations (and somewhat different prevalence rates in more homogenous Asian and African populations), so-called low $\left(C_{G} G_{n<26}\right)$ mutations of the fragile $X$ mental retardation 1 (FMR1) gene, likely, represents the most common known risk factor, including history-based risk factors from medical, genetic and family histories.

Conclusions: Women so affirmatively diagnosed with POS at relative young ages, then have the opportunity to reconsider their reproductive planning and/or choose fertility preservation via oocyte or ovarian tissue cryopreservation at ages when such procedures are clinically much more effective and, therefore, also more cost-effective. Appropriate validation studies will have to precede widespread utilization of this paradigm.

Keywords: Premature ovarian senescence (POS), Occult primary ovarian insufficiency (OPOI), Fertility counseling, Reproductive planning, Functional ovarian reserve, Fragile $X$ mental retardation (FMR1) gene

\section{Background}

The concept of fertility preservation entered medical consciousness primarily through the field of oncology, where increasingly successful chemo- and radiation therapies have improved long-term survival of young cancer patients but often result in premature ovarian senescence (POS) and indeed, frequently in outright premature ovarian failure (POF), also called primary ovarian insufficiency (POI) [1]. Healthy women, delaying childbirth for social reasons, have recently also more actively been pursuing fertility preservation (i.e., "social fertility preservation"), motivated by concerns about inadequate

\footnotetext{
* Correspondence: ykizawa@thechr.com

'The Center for Human Reproduction, 21 East 69th Street, New York, NY 10021, USA

${ }^{2}$ The Foundation for Reproductive Medicine, 21 East 69th Street, New York, NY 10021, USA
}

\section{Biomed Central}

functional ovarian reserve (FOR) by the time they will socially be ready for conception $[2,3]$.

A large pool of patients in need of potential fertility preservation, the approximately $10 \%$ of women who suffer from spontaneously occurring POS, have so far, however, escaped professional attention, quietly and mostly undiagnosed progressing in their POS until becoming clinically symptomatic at advanced stages of low functional ovarian reserve (LFOR) [4]. These women in a large majority of cases suffer from premature ovarian aging (POA), frequently also called occult primary ovarian insufficiency (OPOI), and in approximately only $1 \%$ of cases from POF/POI. Their presenting symptom is usually clinical infertility.

Earlier recognition of being at risk for POA/OPOI and/or POF/POI would offer this patient population the opportunity to preempt late diagnoses at already overt 
infertility stages and, by either changing pregnancy timing or utilizing fertility preservation techniques, would give these young women "at risk" significant opportunities at prevention of later infertility. Earlier recognition of being "at risk" can be based on historical and clinical (laboratory tests) risk factors.

Causes of POS are limited and predictable (Table 1). Only the LFOR, at times associated with endometriosis, has in the literature been proposed as a potential indication for early FOR screening [5]. Other causes of LFOR have so far failed to attract attention. Since a large majority of women affected by POS is not recognized to suffer from LFOR until presentation with infertility, these women at that point often require costly infertility

Table 1 Known risk factors for premature ovarian senescence (POA)

\begin{tabular}{|c|c|}
\hline Medical history & In association with \\
\hline \multirow{2}{*}{$\begin{array}{l}\text { Conditions associated } \\
\text { with low numbers of } \\
\text { follicles at birth/menarche }\end{array}$} & $\begin{array}{l}\text { Turner syndrome - associated with } \\
\text { POF/POI }\end{array}$ \\
\hline & Idiopathic/genetics - association? \\
\hline \multirow[t]{6}{*}{ Excessive recruitment } & FMR1 mutations \\
\hline & $\begin{array}{l}\text { Premutation range }\left(\mathrm{CGG}_{n=55-200}\right) \\
\text { - associated with } \mathrm{POF} / \mathrm{POI}\end{array}$ \\
\hline & $\begin{array}{l}\text { Monoalleleic low sub-genotype - associatec } \\
\text { with POA/OPOI }\end{array}$ \\
\hline & $\begin{array}{l}\text { Biallelic low sub-genotype - associated with } \\
\text { POA/OPOI }\end{array}$ \\
\hline & AMHR2 gene - associated with POF/POI \\
\hline & AIRE gene - associated with POF/POI \\
\hline Other genetic causes & $\begin{array}{l}\text { BRCA1 mutations - associated with POA/ } \\
\text { OPOI }\end{array}$ \\
\hline \multirow{4}{*}{$\begin{array}{l}\text { Space occupying lesions and } \\
\text { latrogenic factors -mostly } \\
\text { associated with POF/POI } \\
\text { but also with POAOPOI }\end{array}$} & Ovarian surgery \\
\hline & Chemotherapy \\
\hline & Radiation therapy \\
\hline & Bone marrow transplantation \\
\hline \multirow[t]{3}{*}{ Other medical risk factors } & Endometriosis - associated with POA/OPOI \\
\hline & $\begin{array}{l}\text { Polycystic ovarian syndrome (PCOS) - } \\
\text { associated with POA/OPOI }\end{array}$ \\
\hline & $\begin{array}{l}>>>>\text { in association with low FMR1 } \\
\text { mutations and risk further augmented in } \\
\text { presence of autoimmunity }\end{array}$ \\
\hline \multirow{6}{*}{$\begin{array}{l}\text { Autoimmunity - mostly } \\
\text { associated with POA/OPOI } \\
\text { but also with POF/POI }\end{array}$} & Thyroid autoimmunity \\
\hline & Adrenal autoimmunity \\
\hline & Any other autoimmunity \\
\hline & Autoimmune polyglandular syndromes \\
\hline & Family history of autoimmune disease* \\
\hline & History of repeated pregnancy loss \\
\hline $\begin{array}{l}\text { Early history of maternal/ } \\
\text { sibling menopause }\end{array}$ & \\
\hline
\end{tabular}

treatments, which with advancing female age, in addition, decrease in effectiveness.

Age-specific follicle stimulating hormone (FSH) and/or anti-Müllerian hormone (AMH) levels have been reported, and allow objective determination of LFOR [4]. Serial longitudinal investigations of FOR in young women "at risk," therefore, should already at relative young ages allow accurate determinations whether patients deviate from normal ovarian aging curves or not.

Absence of such prospective risk assessments in young females is the principal reason why POS is currently still only diagnosed at already advanced clinical stages. Current understanding of POS would, however, at relatively minor costs allow assessments of "risk," and subsequent sequential longitudinal follow up with FOR test parameters like $\mathrm{AMH}$, until final determination whether a patient, indeed, suffers from POS or not. Prospective FOR screening in young "high risk" patients, could, therefore, be viewed as the "PAP smear" for the detection of POS.

Such a diagnostic paradigm very rapidly would permit development of accurate, and appropriately validated, risk prediction models. Reaching similar conclusions, Cil et al. recently made the point that policy makers should integrate oocyte freezing into preventive paradigms for female infertility [6].

\section{Some background on ovarian aging}

Despite suggestions in the literature that ovarian stem cells give rise to fertilizable oocytes [7], current dogma still holds that females are born with a finite pool of follicles/eggs, which rapidly depletes during intrauterine life from a peak ( $\sim$ million follicles/oocytes), which after birth ( 1 million follicles/oocytes) continues to decline at a somewhat slower pace through menarche $(\sim 400,000$ follicles/oocytes) until menopause, when only a few hundred to thousands of follicles/oocytes are left in both ovaries [5]. (Figure 1).

With menarche, menstrual cyclicity is established, when the ovary transforms chaotic follicle recruitment of primordial follicles into regular, usually approximately monthly waves of developing ("growing") follicles. A natural cycle leads to ovulation of only a single dominant follicle/oocyte. All other follicles/oocytes undergo degeneration and apoptosis during the various stages of follicle maturation, many long before the gonadotropin-sensitive stage is reached (Figure 1).

"Ovarian age" is reflected in the total ovarian reserve (TOR) of a patient. The largest part of TOR is made up of still unrecruited, "resting" primordial follicles at very primitive stages of development. "Growing" follicles are a much smaller component of TOR, and represent the socalled FOR. The FOR represents small follicles, which only weeks to months later reach maturity. Ovarian stimulation 


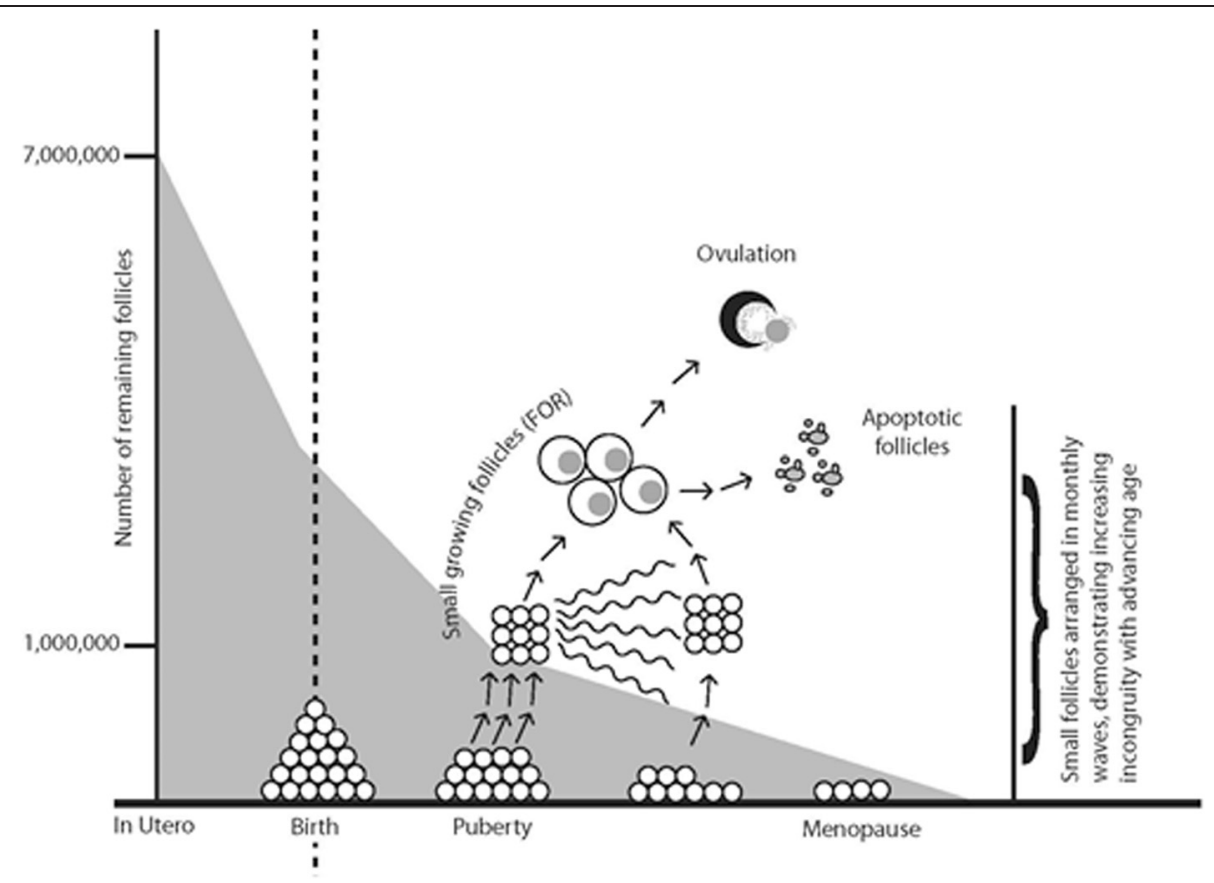

Figure 1 Declining follicle/oocyte numbers with advancing age.

converts the unifollicular natural cycle into a polyfollicular cycle, yielding multiple follicles/oocytes [5].

Genes involved in follicle recruitment appear associated with female age at menopause. Since AMH has been suggested to play a potential role in follicle recruitment, not surprisingly, one such gene controls the AMH type II receptor (AMHR2) [8]. Produced by granulosa cells of small growing follicles, AMH appears to inhibit recruitment as well as subsequent follicle growth $[5,9,10]$.

Another gene holding back recruitment is the AIRE gene. When mutated it leads to rapid follicle depletion and, therefore, POF/POI (see also below) [11]. It also is closely associated with control of immunologic self-tolerance. $\mathrm{Mu}$ tations can lead to breakdown in self-tolerance and autoimmunity, placing this gene at crossroads of autoimmunity and POF/POI [12].

Genes known to affect follicle recruitment, when mutated, blocked or knocked out, primarily lead to rapid depletion of primordial follicles. Genetic control of recruitment, therefore, primarily appears to counteract a natural tendency toward explosive one-time recruitment, as still seen in more primitive water-born species.

Slow recruitment preserves follicles/oocytes at primordial stages, resulting in better TOR and FOR at later ages. This has been demonstrated in association with the FMR1 gene, where so-called low alleles ( $\left.\mathrm{CGG}_{\mathrm{n}<26}\right)$ are associated with rapid depletion [13] and high alleles $\left(\mathrm{CGG}_{\mathrm{n}>34}\right.$ ) preserve richer FOR into advanced female ages [14].

\section{AMH as indicator of FOR}

Speed of follicle recruitment has been reported to correlate with number of remaining primordial follicles. The growing follicle pool, representing FOR, therefore, also correlates with speed of recruitment $[5,9,15]$. AMH reflects this pool of small growing follicles, and is for that reason now widely considered the best laboratory tests to represent TOR $[9,15]$. Sample instability has, however, recently led to questions about reproducibility of results [16]. It appears that with currently commercially available AMH assays, AMH maintains clinically reasonable predictability in women with normal agespecific FOR only up to approximately age 42 . In younger women with OPOI/POA AMH is of clinical value only as long as levels are not extremely low or high [17].

Age-specific AMH levels have been reported by various groups, including ours [18]. Likely the most relevant study to here proposed paradigm is the one by Kelsey and associates, who, via literature searches and own data, accumulated 3,260 data points, which allowed them to define normal AMH levels in healthy premenopausal women of all ages [19]. This large database also included enough females at young ages, where fertility centers usually lack patient populations, to permit determination of prediction limits at various confidence levels (Figure 2).

The authors demonstrated that AMH levels rise from birth, reaching a peak at approximately $24-25$ years. From there on, a developmental stage more familiar to fertility 


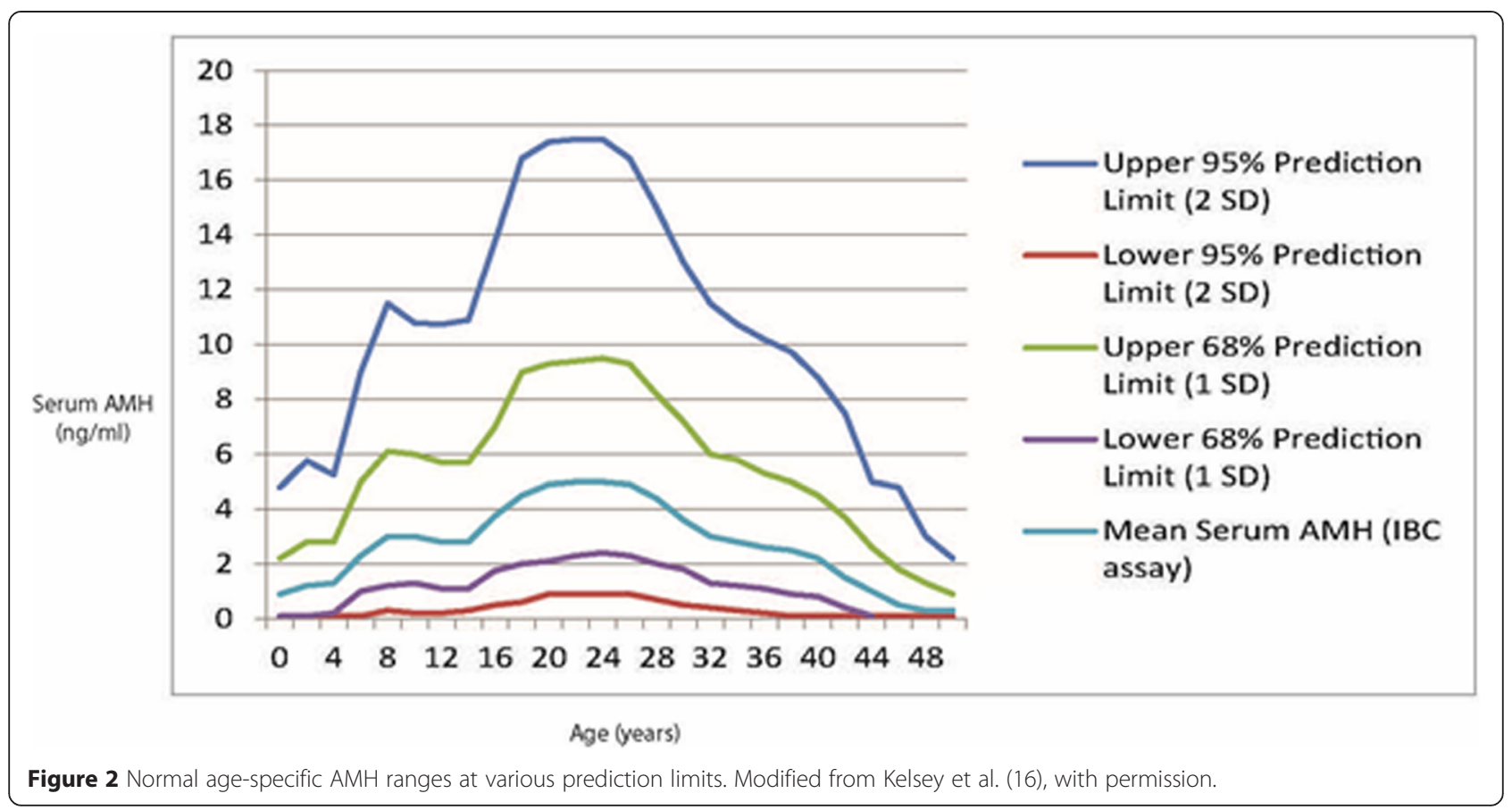

centers, they once again decline [19]. Available data, therefore, now permit determination of what represents normal FOR at young ages, creating the opportunity to assess whether a young female is in normal age-specific range of AMH or not.

$\mathrm{AMH}$, thus, now represents a tool which allows reasonably accurate assessment of FOR and, therefore, in young women, by clinical and laboratory criteria, allows the diagnosis of likely POS (see below for definition). This is done by assessing in young women, previously determined as "at risk," AMH longitudinally, and then establishing whether they follow a normal age-specific ovarian aging curve or not. Those who deviate from normal AMH curves into abnormally low FOR ranges can then, with considerable reliability, at still very young ages can be defined as, indeed, suffering from POS.

\section{Who is at risk for POS?}

Since POS in all of its forms evolves insidiously as a basically asymptomatic process, OPOI/POA and POI/POF diagnoses, as noted before, are currently mostly only made at relatively late stages. Based on well-known risk factors, POS is, however, to varying degrees predictable in its occurrence (Table 1). While the statistical weight of individual predictive risk factors remain to be determined, and likely will vary in different races, as well demonstrated by the varying prevalence rates of low FMR1 mutations among Caucasian, African and Asian women, the mere presence of any one risk factor allows for the initial designation of a young woman as "at risk".
How big the risk really is, can then be determined by the patient's longitudinal follow up.

The PAP smear, once again, offers a good gynecologic analogy to the here proposed POS testing paradigm: As in PAP smear screening risk of cytological abnormalities and frequency of required screening is determined by patient history and PAP smear results, the ultimate risk to develop POS will be established by initial risk determination and subsequent sequential AMH testing. Those who deviate from AMH curves over relative short observation periods will be identified as, indeed, likely suffering from POS, while those following normal aging curves can be reassured.

Table 1 summarizes risk factors for POS. Risk can be the consequence of abnormally low follicle numbers at birth and/or menarche, generally believed to reflect genetic effects [4]. Turner syndrome (see also below) represents a fairly typical example, commonly characterized by premature depletion of follicles at still young ages.

POS can also be the consequence of excessive recruitment. We noted before that the AMHR2 and AIRE genes are associated with excessive recruitment. Excessive recruitment has also been reported in association with low $\left(\mathrm{CGG}_{\mathrm{n}<26}\right)$ FMR1 alleles [20]. Biallelic low FMR1 oocyte donors already at very young ages demonstrate abnormally low FOR, while monoalleleic low FMR1 donors at that point still demonstrate normal age-specific FOR. Over a 4-year observation period, however, even monoalleleic low FMR1 donors already deviate in AMH levels from donors lacking low FMR1 alleles [13]. Since low FMR1 alleles are present in approximately $25 \%$ of all U.S. women, this 
population, likely, reflects the single largest risk pool for POS. An additional gene, recently associated with POA/ OPOI, is the BRCA1 gene [21].

Frequently overlooked as risk factor, are autoimmunityassociated conditions, recently reviewed [12]. Autoimmunity to thyroid often runs in parallel with autoimmunity to ovary; in principle, however, any form of autoimmunity can be associated with POA/OPOI or POF/POI. Since autoimmunity is highly familial, a family history of autoimmunity also represents risk towards POS, which may be why age at menopause is highly familial $[22,23]$. Early maternal and sibling ages at menopause, therefore, also should be considered risk factors towards POS. As autoimmunity can also be associated with repeat miscarriages [24], a history of repeated miscarriage should also be considered a risk factor.

Autoimmunity is believed associated at least with onethird of POS cases [25], and has been reported in humans in three distinct circumstances: Autoimmune (lymphocytic) oophoritis is only rarely observed, and practically exclusively only in presence of Addison's disease [26]. Much more frequently, ovaries appear subject to a still poorly defined autoimmune attack, associated with thyroid autoimmunity, anti-adrenal autoimmunity and other, often non- organ-specific, autoimmune responses [26]. The increasing recognition of the $\mathrm{X}$ chromosome as an "autoimmune chromosome" [27] also explains the very high prevalence of associated autoimmunity with previously noted Turner syndrome $[28,29]$.

Likely, the best-defined form of autoimmune-associated POS occurs with one of the four known autoimmune polyglandular syndromes (APS), so-called APS-1, also known as the polyendocrinopathy candidiasis ectodermal dystrophy or Whitaker syndrome. It is caused by a mutation in the previously noted autoimmune regulator (AIRE) gene [11]. This gene is of importance in the thymus, where it regulates self-tolerance from $\mathrm{T}$ cell attacks. Mutations in the gene, therefore, have been associated with attacks against "self", i.e., autoimmunity.

A gene knockout mouse model (AIRE -/-) demonstrated early follicle depletion, by age 20 weeks, leading in 50-60\% of animals to complete follicle depletion (POF/POI). The AIRE gene, therefore, is the first gene established to be associated with autoimmune-induced POS [11,30].

Other medical factors, including endometriosis and PCOS, can also suggest risk for POS. Endometriosis can cause space-occupying lesions in ovaries, frequently the target of surgeries, which reduce FOR. Endometriosis, however, is also frequently associated with autoimmunity [31]. Similarly, PCOS can be associated with autoimmunity [12], and with the previously noted low FMR1 alleles, by themselves associated with POS [20].

Each in Table 1 listed risk factors, independently, should be considered an indication for serial FOR evaluations of young women until "risk" is either confirmed or refuted.

\section{A prospective screening program}

What differentiates here presented screening paradigm from current practice are two crucial innovations: (i) Identification of high-risk females at very young ages (18-25 years); and (ii) Options for females confirmed as "at risk" to change their pregnancy timing and/or pursue fertility preservation.

The paradigm shift, therefore, is based on a three-step process: (i) identification of young females at increased risk towards POS via FMR1 screening and other risk factors (ii) confirmation or refutation of increased risk by serial longitudinal monitoring with AMH (or other FOR parameters); and (iii) as early counseling and intervention as possible in cases where POS has been confirmed.

It is currently still unknown what percentage of young "high risk" females in such a screening process will be confirmed in a diagnosis of POS. Considering an approximate $10 \%$ prevalence of POS in the general population, the number of affected females should, however, be considerable. Multiple risk factors in one person can be assumed to multiply overall risk, though this, too, awaits clinical confirmation in validation studies.

Figure 3 summarizes the proposed paradigm, and proposes a specific program for validation. While AMH results are generally considered steady [32], AMH levels can also be influenced by hormonal contraceptives [32], and do not appear as stable throughout the menstrual cycle as initially reported [33-35]. We, therefore, recommend as initial screening step two consecutive AMH evaluations, approximately one month apart.

If a patient demonstrates AMH values in age-specific mid-range, annual follow up testing should suffice. Timing can be coordinated with annual gynecological examinations, which, likely, can be switched to biannual testing after 3-5 years of no observed deviations from normal aging curves. Serial testing can, likely, be stopped the latest after approximately 10 years of follow up if no deviations from standard age-specific FOR curves have been observed. Future validation studies should quickly determine appropriate time intervals between tests and overall length of required serial testing.

Low-normal age-specific AMH values at initial evaluation or levels already outside of $95 \%$ confidence intervals for age reflect a likely POS diagnosis, requiring every 3-6 months follow up testing until, with persistently low AMH levels, a final diagnosis of POS can be reached with certainty. Abnormally high levels, often suggest polycystic ovary syndrome (PCOS) $[9,15,16]$. PCOS, in association with low FMR1 alleles, also suggests increased risk of antiovarian autoimmunity [20] and risk towards a quickly depleting ovarian phenotype and subsequent POS [20,36]. 


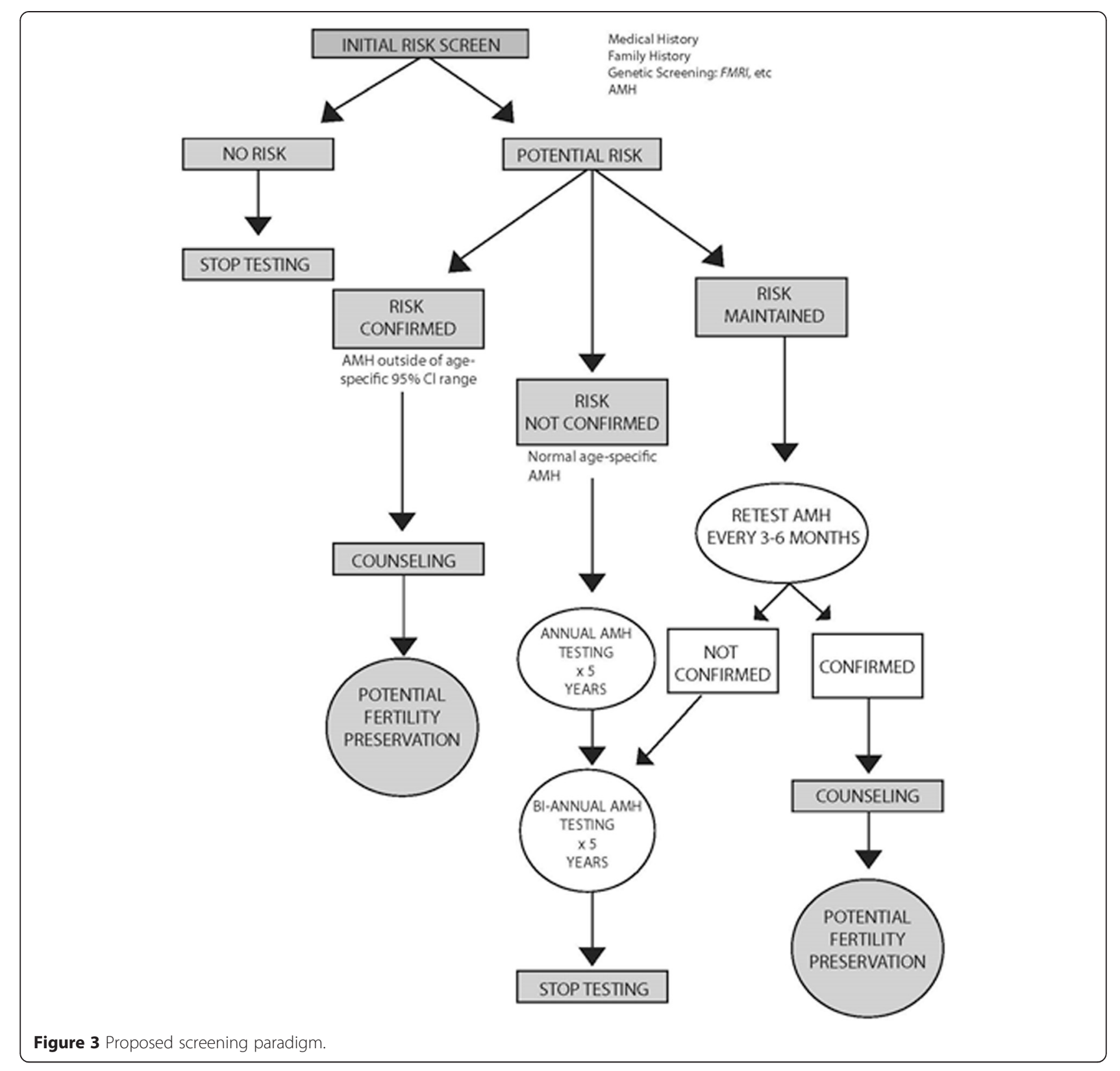

Though POS is usually a slowly progressing process, in a minority of patients, and mostly among those with autoimmune etiology, it can proceed rapidly.

\section{Validation and cost effectiveness}

As noted before, here proposed screening paradigm, of course, requires further validation in clinical practice. Of particular importance is the validation of individual risk factors since they, of course, will greatly vary in their respective individual impacts on risk. Because all of these validations can only be done prospectively over time, and will have to involve study populations of considerable size, they unquestionably will take time.
At the same time, one, however, also has to consider the risk of continuing to do nothing to detect POS earlier in the approximately 10 percent of women who are destined to develop POS. We would argue that the economic cost of late diagnosis with considerable certainty exceeds any potential screening costs, considering the very significant costs of fertility treatments in women POS, who in a large majority of cases, currently, are only diagnosed at rather advanced ages. In practical terms this means that the question to be asked is not whether a prospective screening process to facilitate earlier diagnosis of POS than is currently possible is cost effective but what the individual components of such a process should be to make it most cost effective. 


\section{Conclusions}

Here presented paradigm, as of this point, suggests prospective screening of selected "high-risk" females at young ages. The Eunice Kennedy Shriver National Institute of Child Health and Human Development (NICHD) and the American Society for Reproductive Medicine (ASRM) recently jointly held a conference "to help advance the state of this science from theory to practice" ("Ovarian Reserve: Regulations and Implications for Women's Health, "San Diego, CA, October 2012) [37]. Here proposed new treatment paradigm offers, in the spirit of this conference, an immediately implementable change to current practice.

Finally, we in a recently accepted publication also raised the related issue of assessing risk toward POS in young women who are planning to start utilization of hormonal contraceptives for the long-term [38]. The rational here is that hormonal contraceptives suppress clinical presentations of declining fertility, like cycle irregularities; but they, in addition, affect AMH assessments [33] and, therefore prevent reliable FOR determinations. This recommendation is based on our clinical experience with a considerable number of patients who, after long periods of hormonal contraception use, suddenly found themselves diagnosed with advanced POA/OPOI or even POF/POI.

\section{Abbreviations \\ AMH: Anti-Müllerian hormone; AMHR2: AMH type II receptor; FOR: Functional ovarian reserve; OPOI: Occult primary ovarian insufficiency; OR: Ovarian reserve; POA: Premature ovarian aging; POF: Premature ovarian failure; POI: Primary ovarian insufficiency; POS: Premature ovarian senescence; TOR: Total ovarian reserve.}

\section{Competing interests}

All 3 authors received in the past research support, travel reimbursements and speaker fees from various pharmaceutical companies and/or medical device companies, though none relate to her discussed subjects. V.A. K. has no other potential conflicts to report. N.G. and D.H.B. are listed as co-owners of a number of already awarded and still pending U.S. patents. Awarded patents relate to beneficial therapeutic effects of androgen supplementation on female infertility in women with low ovarian reserve. Pending patents relate to diagnostic claims that the FMR1 gene can be utilized to assess ovarian aging patterns, and to predict fertility treatment chances in association with IVF. N.G. is a shareholder in Fertility Nutraceuticals, LLC, and owner of the CHR-NY. N.G. and D.H.B receive patent royalties from Fertility Nutraceuticals, LLC.

\section{Authors' contributions}

NG. perceived of the subject, performed the literature search and wrote the manuscript with considerable input from VAK. and DHB. at all stages of the process, including manuscript revisions. All authors read and approved the final manuscript.

\section{Acknowledgement}

This project was funded by salary support from The Center for Human Reproduction - New York (CHR-NY).

Received: 18 September 2014 Accepted: 4 April 2015

Published online: 18 April 2015

\section{References}

1. Waimev KE, Duncan FE, Su HI, Smith K, Wallach H, Jona K, et al. Future directions in oncofertility and fertility preservation: a report from the 2011 oncofertility consortium conference. J Adolesc Young Adult Oncol. 2013;2:25-30.
2. Donnez J. Introduction: Fertility preservation, from cancer to benign disease to social reasons: the challenge of the present decade. Fertil Steril. 2013;99:1467-8.

3. Cobo A, Garcia-Velasco JA, Domingo J, Remohl J, Pellicer A. Is vitrification of oocytes useful for fertility preservation for age-related fertility decline and in cancer patients? Fertil Steril. 2013;99:1485-95.

4. Gleicher N, Weghofer A, Barad DH. Defining ovarian reserve to better understand ovarian aging. Reprod Biol Endocrinol. 2011;9:23.

5. Bedoschi G, Turan V, Oktay K. Fertility preservation options in women with endometriosis. Minerva Gynecol. 2013;65:99-103.

6. Cil AP, Bang H, Oktay K. Age-specific probability of live birth with oocyte preservation: an individual patient data metaanalysis. Fertile Steril. 2013;100:492-9. doi: 10./j.fertnstert.2013.04.023

7. Dunlop CE, Telfer EE, Anderson RA. Ovarian stem cells - potential roles in infertility treatment and fertility preservation. Maturitas. 2013;76:279-83.

8. Voorhuis M, Broekmans FJ, Fauser BC, Onland-Moret NC, van der Schouw $Y T$. Genes involved in initial follicle recruitment may be associated with age at menopause. J Clin Endocrinol Metab. 2011;96:E473-9.

9. Ledger WL. Clinical utility of measurement of anti-mullerian hormone in reproductive endocrinology. J Clin Endocrinol Metab. 2010;95:5144-54.

10. Gleicher N, Weghofer A, Barad DH. The role of androgens in follicle maturation and ovulation induction: friend or foe of infertility treatment? Reprod Biol Endocrinol. 2011;9:116.

11. Michels AW, Gottlieb PA. Autimmune polyglandular syndromes. Nat Rev Endocrinol. 2010;6:270-7.

12. Sen A, Kushnir VA, Barad DH, Gleicher N. Endocrne autoimmune diseases and female infertility. Nat Rev Endocrnol. 2014;10:37-50.

13. Kushnir VA, Himaya E, Barad DH, Weghofer A, Gleicher N. Functional ovarian reserve assessment in young oocyte donors based on FMR1 genotypes and sub-genotypes. Fertil Steril. 2013;100:S114.

14. Gleicher N, Weghofer A, Kim A, Barad DH. The impact in older women of ovarian FMR1 genotypes and sub-genotypes on ovarian reserve. PLoS One. 2012; $7:$ :33638.

15. Nelson SM, Anderson RA, Broekmans FJ, Raine-Fenning N, Fleming R, La Marca A. Anti-Müllerian hormone: clairvoyance or crystal clear? Hum Reprod. 2012;27:631-6.

16. Fleming $\mathrm{R}$, Nelson SM. Reproducibility of AMH. Hum Reprod. 2012;27:3639-41.

17. Weghofer A, Dietrich W, Barad DH, Gleicher N. Live birth chances in women with extremely low serum anti-Mullerian hormone levels. Hum Reprod. 2011;26:1905-9.

18. Barad DH, Weghofer A, Gleicher N. Utility of age-specific serum anti-Müllerian hormone concentrations. Reprod Biomed Online. 2011;22:284-91.

19. Kelsey TW, Wright P, Nelson SM, Anderson RA, Wallace WHB. A validated model of serum anti-Müllerian hormone from conception to menopause. PLoS One. 2011;6:e22024.

20. Gleicher N, Weghofer A, Lee $H$, Barad DH. FMR1 genotype with autoimmune-associated polycystic ovary-like phenotype and decreased pregnancy chance. PLoS One. 2010;5:e15303.

21. Oktay K, Kim JY, Barad D, Babayef SN. Association of BRCA1 mutations with occult primary ovarian insufficiency: a possible explanation for the link between infertility and breast/ovarian cancer risk. J Clin Oncol. 2010;28:240-4.

22. van Asselt KM, Kok HS, Pearson PL, Dubas JS, Peeters PH, Te Velde ER, et al. Heritability of menopausal age in mothers and daughters. Fertil Steril. 2004;82:1348-51.

23. Morris DH, Jones ME, Schoemaker MJ, Ashworth A, Swerdlow AJ. Familial concordance for age at natural menopause; results from the breakthrough generations study. Menopause. 2011;18:956-61.

24. Sunkara SK, Khalaf Y, Maheshwari A, Seed P, Coomarasamy A. Association between response to ovarian stimulation and miscarriage following IVF: an analysis of 124,351 IVF pregnancies. Hum Reprod. 2014;29:1218-24.

25. Gleicher N, Weghofer A, Oktay K, Barad D. Do etiologies of premature ovarian aging (POA) mimic those of premature ovarian failure (POF)? Hum Reprod. 2009;24:2395-00.

26. Hoek A, Schoemaker J, Drexhage HA. Premature ovarian failure and ovarian autoimmunity. Endocr Rev. 1997;18:107-34.

27. Bianchi I, Lleo A, Gershwin ME, Invernizzi P. The X chromosome and immune associated genes. J Autoimmun. 2012;38:J187-92.

28. Bukalov VK, Gutin L, Cheng CM, Zhou J, Sheth P, Shah K, et al. Autoimmune disorders in women with turner syndrome and women with karyotypically normal primary ovarian insufficiency. J Autoimmun. 2012;38:315-22. 
29. Lleo A, Moroni L, Caliari L, Invernizzi P. Autoimmunity and Turner's syndrome. Autoimmune Rev. 2012;11:A538-43.

30. Cushman RA. Evidence that the autoimmune regulator gene influences thymic production of ovarian antigens and prevents autoimmune -mediated premature reproductive senescence. Biol Rprod. 2012;86:109.

31. Gleicher N, El Roeiy A, Confino E, Friberg J. Is endometriosis and autoimmune disease? Obstet Gynecol. 1987;70:115-22.

32. Anderson RA, Nelson SM, Wallace WH. Measuring anti-Müllerian hormone for the assessment of ovarian reserve: when and for whom is it indicated? Maturitas. 2012;71:28-33.

33. Kallio S, Puurunen J, Ruokonen A, Vaskivuo T, Piltonen T, Tapanainen JS. Antimüllerian hormone levels decrease in women using combined contraception independently of administered route. Fertil Steril. 2013;99:1305-10

34. Robertson DM, Hale GE, Fraser IS, Hughes CL, Burger HG. Changes in serum antimüllerian hormone levels across the ovulatory menstrual cycle in late reproductive age. Menopause. 2011;18:521-4.

35. Hadlow N, Longhurst K, McClements A, Natawala J, Brown SJ, Matson PL. Variation in antimüllerian hormone concentration during menstrual cycle may change the clinical classification of the ovarian response. Fertil Steril. 2013;99:1791-7.

36. Gleicher N, Weghofer A, Lee IH, Barad DH. Association of FMR1 genotypes with in vitro fertilization (IVF) outcomes based on ethnicity/race. PLoS One. 2011;6:e18781.

37. Lamar C, Tayman S, Rebar R, LaBarbera A, Albertini D, Gracia C. Ovarian reserve: regulation and implications for women's health. Proceedings of the 2012 NICHD-ASRM conference. J Assit Reprod Genet. 2013;30:285-92.

38. Kushnir VA, Barad DH, Gleicher N. Ovarian reserve screening prior to contraception? Reprod Biomed Online. 2014 29; pii:S1472-6483 (14) 004222.doi:10.1026/j.rbmo2014.07.013

\section{Submit your next manuscript to BioMed Central and take full advantage of:}

- Convenient online submission

- Thorough peer review

- No space constraints or color figure charges

- Immediate publication on acceptance

- Inclusion in PubMed, CAS, Scopus and Google Scholar

- Research which is freely available for redistribution 